Proceedings

\title{
Carotenoid Profiling of Orange-Coloured Capsicums: In Search of High-Zeaxanthin Varieties for Eye Health ${ }^{\dagger}$
}

\author{
Rimjhim Agarwal 1,*, Hung T. Hong ${ }^{1}$, Alice Hayward ${ }^{2}$, Stephen Harper ${ }^{3}$, Neena Mitter ${ }^{2}$ \\ and Tim J. $\mathrm{O}^{\prime}$ Hare $^{1}$
}

Citation: Agarwal, R.; Hong, H.T.; Hayward, A.; Harper, S.; Mitter, N.; O'Hare, T.J. Carotenoid Profiling of Orange-Coloured Capsicums: In Search of High Zeaxanthin Varieties for Eye Health. Proceedings 2021, 70 84. https://doi.org/10.3390/ foods_2020-07717

Published: 10 December 2020

Publisher's Note: MDPI stays neutral with regard to jurisdictional claims in published maps and institutional affiliations.

Copyright: (c) 2020 by the authors Licensee MDPI, Basel, Switzerland. This article is an open access article distributed under the terms and conditions of the Creative Commons Attribution (CC BY) license (http://creativecommons.org/licenses /by/4.0/).
1 Centre for Nutrition and Food Sciences, Queensland Alliance for Agriculture and Food Innovation, The University of Queensland, Brisbane, QLD 4072, Australia; h.trieu@uq.edu.au (H.T.H.); t.ohare@uq.edu.au (T.J.O.)

2 Centre for Horticultural Sciences, Queensland Alliance for Agriculture and Food Innovation, The University of Queensland, Brisbane, QLD 4072, Australia; a.hayward@uq.edu.au (A.H.); n.mitter@uq.edu.au (N.M.)

3 School of Agriculture and Food Science, The University of Queensland, Brisbane, QLD 4343, Australia; s.harper1@uq.edu.au

* Correspondence: Rimjhim.agarwal@uq.net.au

† Presented at the 1st International Electronic Conference on Food Science and Functional Foods, 10-25 November 2020; Available online: https://foods_2020.sciforum.net/.

\begin{abstract}
Age-related macular degeneration (AMD) is the leading cause of blindness in developed countries, such as Australia. Lutein and zeaxanthin are the only two carotenoids found in the macular region of the eye. Studies have shown that an intake of $10 \mathrm{mg}$ and $2 \mathrm{mg}$ per day of lutein and zeaxanthin, respectively, can reduce the rate of progression of AMD. The supply of these carotenoids can only be met through dietary sources or supplements, as these compounds cannot be synthesised by humans. Although lutein is relatively abundant in dietary sources, zeaxanthin has limited sources. In this study, eight orange and three red capsicum varieties were analysed for their carotenoid profiles by UHPLC-DAD-APCI-MS. It was observed that the principal carotenoid for seven of the orange varieties was zeaxanthin, and capsanthin for the three red varieties. One orange variety, which had a darker orange hue, had capsanthin and violaxanthin as its principal carotenoids instead of zeaxanthin. Zeaxanthin concentration (the principal carotenoid) in the seven orange varieties varied from $2.6 \pm 0.5 \mathrm{mg} / 100 \mathrm{~g}$ to $25.27 \pm 9.4 \mathrm{mg} / 100 \mathrm{FW}$, suggesting that as little as 7 $\mathrm{g}$ of the high-zeaxanthin line could meet the recommended daily dietary intake of $2 \mathrm{mg} /$ person/day.
\end{abstract}

Keywords: carotenoids; zeaxanthin; bell peppers; eye health; age-related macular degeneration; capsicums

\section{Introduction}

Among all the reasons behind blindness in old people in Australia, age-related macular degeneration (AMD) is one of the leading causes [1]. The macula of the eye is the central region of the retina, thus the degeneration of the macula leads to central blindness. At present, about one in seven Australians, or 1.29 million people over the age 50, have some evidence of this disease. It is estimated that approximately $17 \%$ of these people (over 200,000 ) will experience vision impairment. Almost $15 \%$ of Australians over 80 years of age (around 160,000) have vision loss or blindness from AMD [2].

Lutein, zeaxanthin and meso-zeaxanthin (isomer of zeaxanthin) are carotenoid pigments derived from plants that form the macular pigments [3,4]. They play a protective role in shielding the retina from light damage and reducing the progression of AMD [5]. Lutein constitutes $36 \%$, zeaxanthin $18 \%$, and meso-zeaxanthin $18 \%$ of macular pigment [6]. The remaining $20 \%$ is constituted by the oxidative metabolites such as oxo-lutein, (3-hydroxy- 
$\beta, \varepsilon$-carotene-3'-one), epilutein, $\varepsilon, \varepsilon$-carotene-3,3'-dione, and 9- and $13-\mathrm{Z}$ isomers of both lutein and zeaxanthin [3]. The ratios and distributions of the macular pigments are not uniform and can vary widely between individuals. The ratio of lutein: zeaxanthin (including meso-zeaxanthin) is 1:2.4 in the central macula versus 2:1 in the periphery of the human retina [7]. Lutein and zeaxanthin are yellow and orange, respectively, and absorb blue light wavelengths of around $445 \mathrm{~nm}$ and $450 \mathrm{~nm}$ [6].

A dietary intake of $10 \mathrm{mg}$ and $2 \mathrm{mg}$ per day of lutein and zeaxanthin, respectively, has been associated with reduced progression of AMD [8] and as these carotenoids cannot be synthesised by humans, their supply through dietary sources or supplements is vital [9]. Green leafy vegetables such as spinach and kale are good sources of lutein, however, dietary zeaxanthin is much less common, and is limited to a few orange-coloured fruits and vegetables, such as orange capsicums and sweet corn [10].

Various studies have determined the carotenoid content in red-coloured capsicum fruit [11-13] while only a few studies have focused on orange-coloured capsicums [14,15]. Moreover, the carotenoid profile of orange capsicums, which have been identified as a rich source of zeaxanthin, needs further investigation to better understand the possible variation in zeaxanthin concentrations that may occur across different varieties.

This current study is focused on characterising the carotenoid profiles of eight orange and three red capsicum varieties, in order to investigate the differences in carotenoid profiles and zeaxanthin concentration between capsicums of similar appearance.

\section{Materials and Methods}

\subsection{Plant Materials}

Plants were grown from seeds collected from different seed companies (South Pacific Seeds, Seminis, Rijk Zwaan, De Ruiter and Climbing Fig) in Australia. Seeds were sown into seed trays in early August (ambient conditions $12-22{ }^{\circ} \mathrm{C}$ ) and after 12 weeks, seedlings were transplanted into UQ23 potting mix comprising $70 \%$ composted pine bark and $30 \%$ coco peat and other augments in a randomised block design. Basal fertiliser application was done with Osmocote ${ }^{\circledR}$ slow release fertiliser. One hundred and sixty-five plants were grown in a greenhouse at UQ, Gatton, Australia. Capsicum fruits were harvested in January-February $\left(20-30^{\circ} \mathrm{C}\right)$ at the same maturity stage. Five plants per variety were harvested for the carotenoid analysis. For each plant, three fruits were selected and were measured for their hue angles using the Konica Minolta CR-400 (Konica Minolta, Osaka, Japan) chromameter to ensure the same maturity stage.

\subsection{Carotenoid Analysis}

The pericarp of each fruit was dissected in cold room and were milled in the presence of liquid nitrogen. A MM400 Retsch Mixer Mill (Hann, Germany) was operated at $30 \mathrm{~Hz}$ for $60 \mathrm{~s}$ to achieve a fine dry powder. This fine powder $(1 \mathrm{~g}$ each) was then dissolved in 6 $\mathrm{mL}$ ethanol in a $50 \mathrm{~mL}$ Falcon tube and was vortexed for $20 \mathrm{~s}$.

Three milliliters of $\mathrm{NaCl}(5 \%)$ were added to the sample, followed by $10 \mathrm{~mL}$ extraction solvent (20:80 DCM:hexane). The solution was then centrifuged using an Eppendorf centrifuge (models 5804R and 5810R, Hamburg, Germany) at $4000 \mathrm{rpm}$ for $5 \mathrm{~min}$ at $25^{\circ} \mathrm{C}$ to separate layers. The supernatant was transferred to a new $50 \mathrm{~mL}$ Falcon tube and the pellet was re-extracted twice or until the pellet was colourless. Extracted solution was put into a centrifugal evaporator (miVac Duo concentrator Genevac, model DUP-23050-H00, rotor DRC-5-CCT-008, Ipswich, England) fixed at $25-30{ }^{\circ} \mathrm{C}$ for $45 \mathrm{~min}$ or until dry. The carotenoids were then de-esterified using $15 \%$ methanolic $\mathrm{KOH}$ for $10 \mathrm{~min}$ at room temperature in the dark.

The extracted carotenoid samples (saponified and non-saponified) were reconstituted in 50:50 MeOH:MTBE (0.1\% BHT). All the samples were analysed in technical replicates of three to ensure repeatability of the experiment. Five microliters of each sample were injected in the Waters AcquityTM UHPLC-PDA System (Waters, Milford, MA, USA). The mobile solvents were methanol $0.1 \%$ formic acid (mobile phase A) and methyl 
tert-butyl ether (MTBE, mobile B). Column temperature hold was at $25^{\circ} \mathrm{C}$ with a flow rate of $0.6 \mathrm{~mL} / \mathrm{min}$ on a YMC C 30 Carotenoid Column, $3 \mu \mathrm{m}, 4.6 \times 250 \mathrm{~mm}$ (Waters, Milford, MA, USA). The gradient elution of $40 \mathrm{~min}$ was started with isocratic at $20 \%$ B (mobile phase B: $0.1 \%$ formic acid in MTBE) for $1 \mathrm{~min}$, from $20 \% \mathrm{~B}$ to $25 \% \mathrm{~B}$ in $20 \mathrm{~min}$ and $30 \% \mathrm{~B}$ in the next of $10 \mathrm{~min}$ before increasing sharply to $70 \%$ mobile phase B in $3 \mathrm{~min}$, followed by conditioning for $1 \mathrm{~min}$, and re-equilibrating for $6 \mathrm{~min}$.

Identification of lutein, zeaxanthin, $\beta$-carotene, capsanthin, and capsorubin was determined by using external standards and then the UV absorption spectrum [8]. A Shimadzu ultra-high-pressure liquid chromatography- diode array detection- atmospheric pressure chemical ionisation- mass spectrometry (UHPLC-DAD-APCI-MS) system (Shimadzu, Kyoto, Japan, System 3) was used with a Nexera X2 UHPLC system consisting of a system controller (CBM-30A), three pumps (LC-30AD), an auto sampler (SIL-30AC), column heater (CTO-20AC), diode array detector (DAD) (SPD-M30A), and two degassers (DGU-20A3R and DGU-20A5R). The Nexera X2 UHPLC system coupled to an LCMS-8060 triple quadrupole mass spectrometer (Shimadzu, Kyoto, Japan) and the APCI source was used to identify the molecular mass and fragment ions of lutein, zeaxanthin, $\beta$-carotene, capsanthin, capsorubin, and other carotenoids without external standards. The mobile solvents and gradient elution were used the same as the Waters Acquity ${ }^{\mathrm{TM}}$ UHPLC-PDA System. Standard curve dilutions were measured in the UHPLC to identify retention times and peak areas. Lutein, zeaxanthin, capsanthin, $\alpha$ and $\beta$-cryptoxanthin, $\alpha$-carotene, and $\beta$-carotene from the sample extracts were identified and quantified by comparison with the retention times and absorption spectra peaks from standards and their molecular masses and fragment ions.

\section{Results and Discussion}

\subsection{Carotenoid Profile of Varieties}

Figure 1 depicts the difference between the carotenoid profiles of orange and red capsicums varieties. Seven of eight orange-coloured capsicums had similar carotenoid profiles, with zeaxanthin (50-75\%) as their principal carotenoid (BP, 199-9, PS, Orandino, DSP, Daina, and Boogie). Zeaxanthin, an orange-coloured carotenoid, supports the observed orange colour of these varieties. Red capsicums (Hugo, Plato, and Warlock), in contrast, had capsanthin (50-70\%) as their principal carotenoid. The prevalence of red carotenoid pigments (capsanthin and capsorubin) in these varieties supports the observed red colour. Interestingly, the variety "179-8", which was a darker orange hue than the other orange capsicums, had violaxanthin (33\%) and capsanthin (39\%) as its major carotenoids. The mixture of these yellow and red carotenoids, namely violaxanthin and capsanthin, respectively, likely explains the resultant darker hue observed in this variety. 


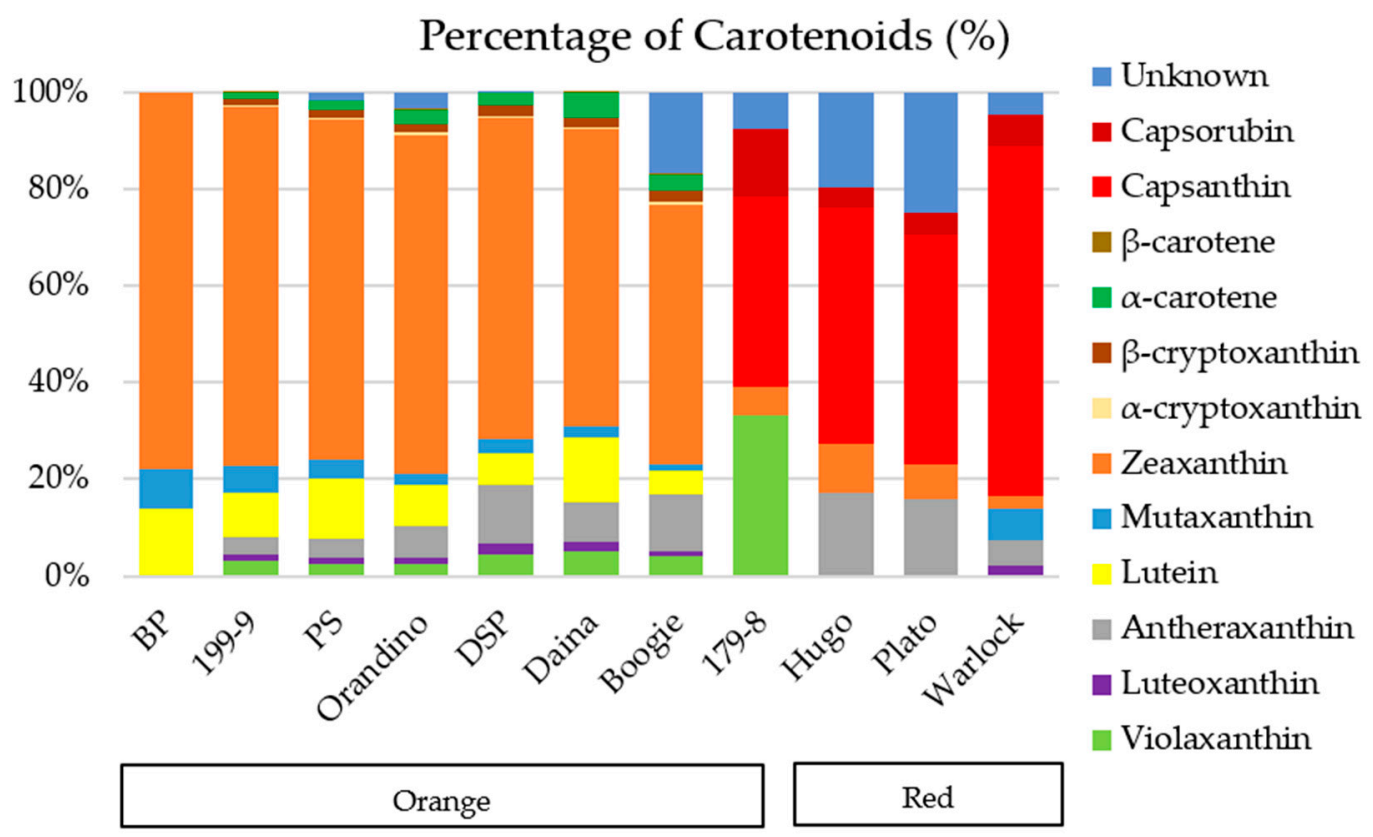

Figure 1. Percentage of carotenoids (after de-esterification) in different orange- and red-coloured capsicums.

Lutein was observed only in the seven orange capsicum varieties, and was absent in the red and dark orange varieties. Similar results for orange and dark orange capsicums have been previously reported by Rodriguez-Uribe et al. [15], with zeaxanthin (45-60\%) being the major carotenoid in orange capsicums, and violaxanthin $(22 \%)$ and capsanthin $(27 \%)$ being the major carotenoids in the dark orange variety. Similar observations of the principal carotenoids were also reported in other studies with regard to orange- and red-coloured capsicums $[12,16,17]$. However, in a separate study conducted by Guzman et al. [14], $\beta$-carotene, an orange-coloured carotenoid, was the principal carotenoid in most of the orangecoloured capsicums. This is in contrast to the current study, where beta-carotene was a minor carotenoid in all varieties assessed. Thus, it can be inferred from the past and present studies that not all orange-coloured capsicums are due to accumulation of zeaxanthin, and other carotenoids such as $\beta$-carotene or a mix of yellow and red carotenoids can also lead to orange colour in capsicums.

\subsection{Zeaxanthin Concentration of Varieties}

From Table 1, it can be calculated that the varieties requiring the smallest amount of tissue to be ingested to meet the daily suggested intake of zeaxanthin are "Orandino" and "Boogie" (nearly $7 \mathrm{~g}$ ), whereas $25 \mathrm{~g}$ and $30 \mathrm{~g}$ tissue would be required to meet the same suggested intake for the "199-9" and "DSP" varieties. Similarly, 260-440 g of tissue would be required with the red capsicums to meet the same daily zeaxanthin requirement. Interestingly, despite its intense orange colour, the dark orange variety "179-8" had the lowest zeaxanthin concentration, so that as much as $900 \mathrm{~g}$ would be required to meet the suggested zeaxanthin intake. Considering an average size of $400 \mathrm{~g}$ of capsicums in the Australian market, this would mean that the consumption of a few slices of high-zeaxanthin orange varieties would be sufficient as compared to a whole capsicum in the case of red lines to meet the recommended zeaxanthin daily dietary intake. 
Table 1. Zeaxanthin concentration (mg/100 g FW) and the tissue needed to meet a daily dietary intake of zeaxanthin of $2 \mathrm{mg} /$ per person/per day.

\begin{tabular}{ccccc}
\hline Variety & Colour & Total Carotenoid Conc. Zeaxanthin Conc. Tissue Needed (g) \\
\hline Orandino & Orange & $40.0 \pm 12.3$ & $28.0 \pm 8.5$ & 7 \\
Boogie & Orange & $50.3 \pm 22.5$ & $27.0 \pm 9.4$ & 7 \\
PS & Orange & $29.1 \pm 13.7$ & $20.5 \pm 10.3$ & 10 \\
Diana & Orange & $22.0 \pm 14.1$ & $13.5 \pm 8.4$ & 15 \\
$199-9$ & Orange & $10.4 \pm 3.2$ & $8.0 \pm 2.7$ & 25 \\
DSP & Orange & $9.3 \pm 1.5$ & $6.2 \pm 1.0$ & 30 \\
BP & Orange & $2.4 \pm 0.1$ & $1.9 \pm 0.1$ & 100 \\
179-8 & Dark orange & $3.7 \pm 1.2$ & $0.2 \pm 0.1$ & 910 \\
Hugo & Red & $7.5 \pm 1.3$ & $0.8 \pm 0.2$ & 260 \\
Plato & Red & $9.5 \pm 2.0$ & $0.7 \pm 0.3$ & 300 \\
Warlock & Red & $17.8 \pm 7.6$ & $0.5 \pm 0.4$ & 440 \\
\hline
\end{tabular}

It is also worth noting that varieties with a high percentage of zeaxanthin (Figure 1) do not necessarily have a high zeaxanthin concentration, for instance, variety "BP" has the highest percentage of zeaxanthin $(80 \%)$ among all the varieties but its zeaxanthin concentration is only $1.9 \pm 0.1 \mathrm{mg} / 100 \mathrm{~g} \mathrm{FW}$, which is significantly less than the highest zeaxanthin concentration of "Orandino" and "Boogie" (28.0 \pm 8.5 and $27.0 \pm 9.4 \mathrm{mg} / 100 \mathrm{~g}$ $\mathrm{FW}$, respectively). This observation suggests that zeaxanthin production relative to $\mathrm{FW}$, not relative to total carotenoids produced, is an important consideration in the dietary value of various cultivars.

\section{Conclusions}

From the present study, it can be concluded that although orange capsicums are a considerably better source of zeaxanthin compared to red capsicums, and had similar carotenoid profiles to each other, there was still considerable variation in zeaxanthin concentration between the orange varieties. It is also worth noting that orange colour does not necessarily indicate high zeaxanthin concentration, for instance, in the dark orange variety investigated in this study, it was due to a mixture of red (capsanthin and capsorbin) and yellow (violaxanthin) carotenoids. Thus, depending on the zeaxanthin concentration, different capsicum varieties will have different dietary value in meeting the suggested daily dietary requirement of zeaxanthin.

Funding: This study was funded in part by "Naturally Nutritious" (Hort Innovation, project HN15001).

Institutional Review Board Statement: Not applicable.

Informed Consent Statement: Not applicable.

Data Availability Statement: Data is contained within the article or supplementary material.

\section{References}

1. Keel, S.; Xie, J.; Foreman, J.; Van Wijngaarden, P.; Taylor, H.R.; Dirani, M. Prevalence of Age-Related Macular Degeneration in Australia: The Australian National Eye Health Survey. JAMA Ophthalmol. 2017, 135, 1242-1249, doi:10.1001/jamaophthalmol.2017.4182.

2. About Macular Degeneration. Available online: https://www.mdfoundation.com.au/content/what-is-macular-degeneration (accessed on 14 October 20202).

3. Arunkumar, R.; Gorusupudi, A.; Bernstein, P.S. The macular carotenoids: A biochemical overview. Biochim. Biophys. Acta BBA Mol. Cell Biol. Lipids 2020, 1865, 158617, doi:10.1016/j.bbalip.2020.158617.

4. Abdel-Aal, E.-S.M.; Akhtar, H.; Zaheer, K.; Ali, R. Dietary Sources of Lutein and Zeaxanthin Carotenoids and Their Role in Eye Health. Nutrients 2013, 5, 1169-1185, doi:10.3390/nu5041169. 
5. Tosini, G.; Ferguson, I.; Tsubota, K. Effects of blue light on the circadian system and eye physiology. Mol. Vis. 2016, 22, 61-72.

6. Landrum, J.T.; Bone, R.A. Lutein, zeaxanthin, and the macular pigment. Arch. Biochem. Biophys. 2001, 385, 28-40.

7. Bone, R.A.; Landrum, J.T.; Fernandez, L.; Tarsis, S.L. Analysis of the macular pigment by HPLC: Retinal distribution and age study. Investig. Ophthalmol. Vis. Sci. 1988, 29, 843-849.

8. Chew, E.Y.; Clemons, T.E.; SanGiovanni, J.P.; Danis, R.P.; Ferris, F.L.; Elman, M.J.; Antoszyk, A.N.; Ruby, A.J.; Orth, D.H.; Bressler, S.B.; et al. Secondary Analyses of the Effects of Lutein/Zeaxanthin on Age-Related Macular Degeneration Progression. JAMA Ophthalmol. 2014, 132, 142-149, doi:10.1001/jamaophthalmol.2013.7376.

9. Perera, C.O.; Yen, G.M. Functional Properties of Carotenoids in Human Health. Int. J. Food Prop. 2007, 10, 201-230, doi:10.1080/10942910601045271.

10. Perry, A.; Rasmussen, H.; Johnson, E.J. Xanthophyll (lutein, zeaxanthin) content in fruits, vegetables and corn and egg products. J. Food Compos. Anal. 2009, 22, 9-15, doi:10.1016/j.jfca.2008.07.006.

11. Ha, S.-H.; Kim, J.-B.; Park, J.-S.; Lee, S.-W.; Cho, K.-J. A comparison of the carotenoid accumulation in Capsicum varieties that show different ripening colours: Deletion of the capsanthin-capsorubin synthase gene is not a prerequisite for the formation of a yellow pepper. J. Exp. Bot. 2007, 58, 3135-3144, doi:10.1093/jxb/erm132.

12. Kim, J.-S.; An, C.G.; Park, J.-S.; Lim, Y.P.; Kim, S. Carotenoid profiling from 27 types of paprika (Capsicum annuum L.) with different colors, shapes, and cultivation methods. Food Chem. 2016, 201, 64-71, doi:10.1016/j.foodchem.2016.01.041.

13. Mínguez-Mosquera, M.I.; Hornero-Méndez, D. Changes in Carotenoid Esterification during the Fruit Ripening of Capsicum annuum Cv. Bola. J. Agric. Food Chem. 1994, 42, 640-644, doi:10.1021/jf00039a007.

14. Guzman, I.; Hamby, S.; Romero, J.; Bosland, P.W.; O'Connell, M.A. Variability of carotenoid biosynthesis in orange colored Capsicum spp. Plant Sci. 2010, 179, 49-59, doi:10.1016/j.plantsci.2010.04.014.

15. Rodriguez-Uribe, L.; Guzman, I.; Rajapakse, W.; Richins, R.D.; O'Connell, M.A. Carotenoid accumulation in orange-pigmented Capsicum annuum fruit, regulated at multiple levels. J. Exp. Bot. 2011, 63, 517-526, doi:10.1093/jxb/err302.

16. Bonaccorsi, I.; Cacciola, F.; Utczas, M.; Inferrera, V.; Giuffrida, D.; Donato, P.; Dugo, P.; Mondello, L. Characterization of the pigment fraction in sweet bell peppers (Capsicum annuum L.) harvested at green and overripe yellow and red stages by offline multidimensional convergence chromatography/liquid chromatography-mass spectrometry. J. Sep. Sci. 2016, 39, 3281-3291, doi:10.1002/jssc.201600220.

17. Minguez-Mosquera, M.I.; Hornero-Mendez, D. Formation and transformation of pigments during the fruit ripening of Capsicum annuum cv. Bola and Agridulce. J. Agric. Food Chem. 1994, 42, 38-44. 